\title{
Frequency Identification of Wiener Systems with Backlash Operators using Separable Least Squares Estimators
}

\author{
F. Giri *, Y. Rochdi *, F. Ikhouane**, A. Brouri*, F.Z. Chaoui* \\ *Université de Caen Basse-Normandie, GREYC UMR CNRS, 14032 Caen, France (fouad.giri@unicaen.fr). \\ **Universitat Politecnica de Catalunya, Barcelona, Spain (e-mail: faycal.ikhouane@upc.edu)
}

\begin{abstract}
This paper deals with the identification of Wiener models that involve backlash operators bordered by possibly noninvertible parametric lines. The latter are also allowed to cross each other making possible to account for general-shape static nonlinearities. The linear dynamic subsystem is not-necessarily parametric but is BIBO stable. A frequency identification method is developed that provides estimates of the nonlinear operator parameters as well as estimates of the linear subsystem frequency gain. The method involves standard and separable least squares estimators that all are shown to be consistent. Backlash operators and memoryless nonlinearities are handled within a unified framework.
\end{abstract}

\section{INTRODUCTION}

Wiener models consist of a linear dynamic block, followed in series by a nonlinear static operator. Despite its apparent simplicity, this model structure features a high modeling capability formally established and practically confirmed in various application areas. In the case of parametric Wiener systems, the identification problem has been dealt with following different approaches including half-substitution iterative technique (Vörös, 2001), subspace and separable nonlinear least-squares methods (Westwick, 1996; Bruls et al., 1999), output error and maximum likelihood algorithms (e.g.Vanbaylen et al., 2008; Wills and Ljung, 2010). Nonparametric Wiener systems have mainly been dealt with using stochastic methods (e.g. Greblicki and Pawlak, 2008; Chen, 2006; Hu et al., 2005; Mzyk, 2010) and frequency methods (e.g. Giri et al., 2009; Bai and Reyland, 2009). Most previous works have focused on Wiener systems with memoryless nonlinearities. The case of memory nonlinearities has recently been dealt with in (Giri et al., 2010) where a frequency identification method has been developed for Wiener systems including backlash operators bordered with straight lines. In this paper, Wiener system identification is addressed in the case where the nonlinear element is a parametric backlash operator bordered with polynomial lines. Furthermore, the bordering lines are presently allowed to be noninvertible and crossing (Fig. 1). This feature makes possible to handle, within a unified framework, backlash operators and static nonlinearities (as the latter may be viewed as backlash with identical ascendant and descendent borders). The considered class of backlash operators may naturally exist in engineering, economy and biology systems. It may also arise when static nonlinearities are connected in series with standard backlash operators (Giri et al., 2010). A frequency-domain identification method is developed that provides accurate estimates of: (i) the linear subsystem frequency gain $G(j \omega)$ which presently is allowed to be nonparametric; (ii) and the coefficients of the nonlinearity bordering polynomials. The identification method involves the application of sine signals and makes use of standard and separable least-squares estimators which all are shown to be consistent.

The paper is organized as follows: the identification problem is formulated in Section 2; a useful frequency analysis is presented in Section 3; the identification method is designed and analyzed in Section 4. For space limitation all proofs are removed, they can be provided by the authors upon request.

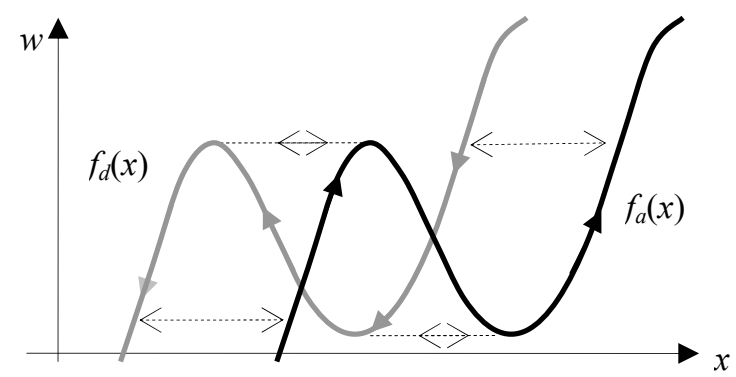

Fig. 1. Backlash operator with noninvertible bordering lines

\section{IDENTIFICATION PROBLEM FORMULATION}

Standard Wiener systems consist of a linear dynamic subsystem $G(s)$ followed in series by a memoryless nonlinear operator $F[$.$] . Presently, both memory and$ memoryless nonlinearities are handled within a unified framework. Analytically, the Wiener system is described by:

$$
\begin{array}{lll}
x(t)=g(t) * u(t) & \text { with } & g(t)=\mathfrak{L}^{-1}(G(s)) \\
y(t)=w(t)+\xi(t) & \text { with } & w(t)=F[x](t)
\end{array}
$$

where $u(t)$ and $y(t)$ denote the system input and output; $x(t)$ and $w(t)$ are not accessible to measurement; the noise $\xi(t)$ it is a stationary ergodic sequence of zero-mean independent random variables. In (1), the symbol $*$ refers to 
signal convolution and $\mathfrak{L}$ to Laplace transform. Accordingly, $G(s)$ is a transfer function, with impulse response $g(t)$, representing the system dynamics. Presently, $G(s)$ is allowed to be nonparametric and infinite order but $g \in L_{1}$ so that $G(s)$ is BIBO stable. The nonlinear operator $F[$.$] is a$ backlash operator characterized by its ascendant and descendant bordering lines, $\left(x, f_{a}(x)\right)$ and $\left(x, f_{d}(x)\right)$. The latter are possibly noninvertible and crossing but parametric. To fix idea, these are assumed to be polynomials:

$$
f_{a}(x)=a_{0}+a_{1} x+\ldots+a_{n} x^{n}, f_{d}(x)=d_{0}+d_{1} x+\ldots+d_{n} x^{n}
$$

The integer $n$ is known but the coefficient vectors $\left[\begin{array}{lll}a_{1} & \ldots & a_{n}\end{array}\right]^{T}$ and $\left[\begin{array}{lll}d_{1} & \ldots & d_{n}\end{array}\right]^{T}$ are not; these are only assumed to be nonzero. The memory element $F[$.] operates as follows:

- If the working point $(x(t), w(t))$ is moving on the ascendant (resp. descendant) border then, it will keep moving on that border as long as $\dot{x}(t) \geq 0$ (resp. $\dot{x}(t) \leq 0$ ).

- If the derivative $\dot{x}(t)$ changes sign, while the working point $(x(t), w(t))$ was moving on one border, then it leaves the current border and keeps on moving horizontally towards the opposite border. When it reaches the opposite border, it moves along it as long as $\dot{x}(t)$ does not change sign.

Static nonlinearities (i.e. $\left.f_{a}()=.f_{d}()=.f().\right)$ are handled, together with backlash operators, within a unified treatment. The identification problem consists in determining accurate estimates of the nonlinear operator parameters $\left(a_{i}, d_{i} ; i=1 \ldots n\right)$ and the linear frequency gain $G\left(j \omega_{k}\right)$ with $\omega_{k}(k=1 \ldots m)$ appropriately chosen.

\section{FREQUENCY-DOMAIN ANALYSIS}

Useful Model Expressions. When the system (1)-(2) is excited with $u(t)=U \cos (\omega t)$, it generates in steady state the signals:

$$
\begin{aligned}
& x_{U, \omega}(t)=U|G(j \omega)| \cos (\omega t-\varphi(\omega)) ; \varphi(\omega)=-\angle G(j \omega) \\
& w_{U, \omega}(t)=F\left[x_{U, \omega}\right](t), \quad y_{U, \omega}(t)=w_{U, \omega}(t)+\xi(t)
\end{aligned}
$$

Then, it follows from (4a-b) that the undisturbed output $w_{U, \omega}(t)$ is a periodic signal with period $2 \pi / \omega$. Now, let

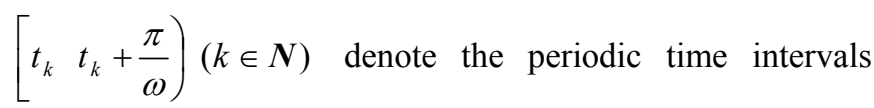
where $\cos (\omega t-\varphi(\omega))$ is decreasing (or, equivalently $\sin (\omega t-\varphi(\omega)) \geq 0)$. Then, by definition of the operator $F[$.$] ,$ there is a pair of real numbers $\left(\tau_{a}, \tau_{d}\right)$, with $0 \leq \tau_{a}<\pi / \omega$ and $0 \leq \tau_{d}<\pi / \omega$, so that (see Figs $2 \mathrm{a}-\mathrm{b}$ ):

$t_{k}-t_{k-1}=2 \pi / \omega$, for all $k \in N$

$w_{U, \omega}(t)=f_{a}\left(x_{U, \omega}\left(t_{k}\right)\right)=$ const., for $t \in\left[\begin{array}{ll}t_{k} & \left.t_{k}+\tau_{d}\right)\end{array}\right.$

$w_{U, \omega}(t)=f_{d}\left(x_{U, \omega}(t)\right), \quad$ for $t \in\left[t_{k}+\tau_{d} t_{k}+\frac{\pi}{\omega}\right)$

$$
\begin{aligned}
& w_{U, \omega}(t)=f_{d}\left(x_{U, \omega}\left(t_{k}+\frac{\pi}{\omega}\right)\right) ; \quad t \in\left[t_{k}+\frac{\pi}{\omega} t_{k}+\frac{\pi}{\omega}+\tau_{a}\right) \\
& w_{U, \omega}(t)=f_{a}\left(x_{U, \omega}(t)\right), \quad t \in\left[t_{k}+\frac{\pi}{\omega}+\tau_{a} t_{k}+\frac{2 \pi}{\omega}\right) \\
& f_{a}(U|G(j \omega)|)=f_{d}(U|G(j \omega)|) \Leftrightarrow \tau_{d}=0 \\
& f_{a}(-U|G(j \omega)|)=f_{d}(-U|G(j \omega)|) \Leftrightarrow \tau_{a}=0
\end{aligned}
$$

On the other hand, the couple $(G, F)$ in not uniquely defined by (1)-(2); any couple of the form $(G(s) / K, F[K x])$ is also a model, whatever $K \neq 0$. But, as long as phase estimation is concerned, all models are identical either to $(G, F)$ or to its dual $\left(G^{-}, F^{-}\right)$defined by:

$G^{-}(s)=-G(s), \quad F^{-}[x]=F[-x]$

$F^{-}$[.] is in turn a backlash operator bordered by the couple of functions $\left(f_{a}^{-}, f_{d}^{-}\right)$with:

$\begin{array}{ll}f_{a}^{-}(x) \stackrel{\text { def }}{=} f_{d}(-x)=\sum_{i=0}^{n} a_{i}^{-} x^{i} & \text { with } a_{i}^{-}=d_{i}(-1)^{i} \\ f_{d}^{-}(x) \stackrel{\text { def }}{=} f_{a}(-x)=\sum_{i=0}^{n} d_{i}^{-} x^{i} & \text { with } d_{i}^{-}=a_{i}(-1)^{i}\end{array}$

Then, equations like (4a-b) can be expressed in term of $\left(G^{-}, F^{-}\right)$because this also is a model. Specifically, one has:

$x_{U, \omega}^{-}(t)=U|G(j \omega)| \cos \left(\omega t-\varphi^{-}(\omega)\right)$

$\varphi^{-}(\omega)=-\angle G^{-}(j \omega)=\pi-\angle G(j \omega)$ (modulo $\left.2 \pi\right)$

$w_{U, \omega}(t)=F^{-}\left[x_{U, \omega}^{-}\right](t) ; y_{U, \omega}(t)=w_{U, \omega}(t)+\xi(t)$

Using (8a-b) and (4a), one gets the relation:

$x_{U, \omega}^{-}(t)=-x_{U, \omega}(t)$

Using (9), properties equivalent to (5a-g) are obtained in function of the model $\left(G^{-}, F^{-}\right)$. In particular, one has:

$$
\begin{aligned}
& w_{U, \omega}(t)=f_{d}^{-}\left(x_{U, \omega}^{-}(t)\right), \quad \forall t \in\left[t_{k}^{-}+\tau_{d}^{-} t_{k}^{-}+\frac{\pi}{\omega}\right), \\
& w_{U, \omega}(t)=f_{a}^{-}\left(x_{U, \omega}^{-}(t)\right), \quad \forall t \in\left[t_{k}^{-}+\frac{\pi}{\omega}+\tau_{a}^{-} t_{k}^{-}+\frac{2 \pi}{\omega}\right),
\end{aligned}
$$

where $k \in N$ and:

$t_{k}^{-} \stackrel{\text { def }}{=} t_{k}+\frac{\pi}{\omega}(k=0,1 \ldots), \quad \tau_{a}^{-} \stackrel{\text { def }}{=} \tau_{d}, \quad \tau_{d}^{-} \stackrel{\text { def }}{=} \tau_{a}$

From the above definitions it is readily seen that:

$\left[t_{k}^{-}+\tau_{d}^{-} t_{k}^{-}+\frac{\pi}{\omega}\right)=\left[t_{k}+\frac{\pi}{\omega}+\tau_{a} t_{k}+\frac{2 \pi}{\omega}\right)$ and

$$
\left[t_{k}^{-}+\frac{\pi}{\omega}+\tau_{a}^{-} t_{k}^{-}+\frac{2 \pi}{\omega}\right)=\left[\begin{array}{ll}
t_{k+1}+\tau_{d} & \left.t_{k+1}+\frac{\pi}{\omega}\right)
\end{array}\right.
$$

Note that, when $w_{U, \omega}(t)=f_{a}^{-}\left(x_{U, \omega}^{-}(t)\right) \quad$ (resp. $\left.w_{U, \omega}(t)=f_{d}^{-}\left(x_{U, \omega}^{-}(t)\right)\right)$ then, $x_{U, \omega}^{-}(t)$ is increasing (resp. decreasing).

System Output Response. Three situations are possible, depending on $U|G(j \omega)|$. The first one, illustrated by Fig. 2, 
corresponds to the case where $f_{a}(U|G(j \omega)|) \neq f_{d}(U|G(j \omega)|)$ and/or $\quad f_{a}(-U|G(j \omega)|) \neq f_{d}(-U|G(j \omega)|)$. Then, $\quad w_{U, \omega}(t)$ exhibits constant stages at instants $t_{k}$ and/or $t_{k}+\pi / \omega$. In this respect, recall that $\varphi(\omega)=\omega t_{k}$ (modulo $\left.2 \pi\right)$. The second situation, illustrated by Fig. 3, is one where $w_{U, \omega}(t)$ is timevarying but $f_{a}(U|G(j \omega)|)=f_{d}(U|G(j \omega)|) \quad$ and $f_{a}(-U|G(j \omega)|)=f_{d}(-U|G(j \omega)|)$. Then, $w_{U, \omega}(t)$ includes no constant stages implying $\tau_{a}=\tau_{d}=0$. The third situation is one where $U|G(j \omega)|$ is too small so that the limit cycle described by the working point $\left(x_{U, \omega}(t), w_{U, \omega}(t)\right)$ reduces to a horizontal segment. Then, $w_{U, \omega}(t)$ turns out to be constant and so is useless. The above remarks are formalized in Proposition 1.
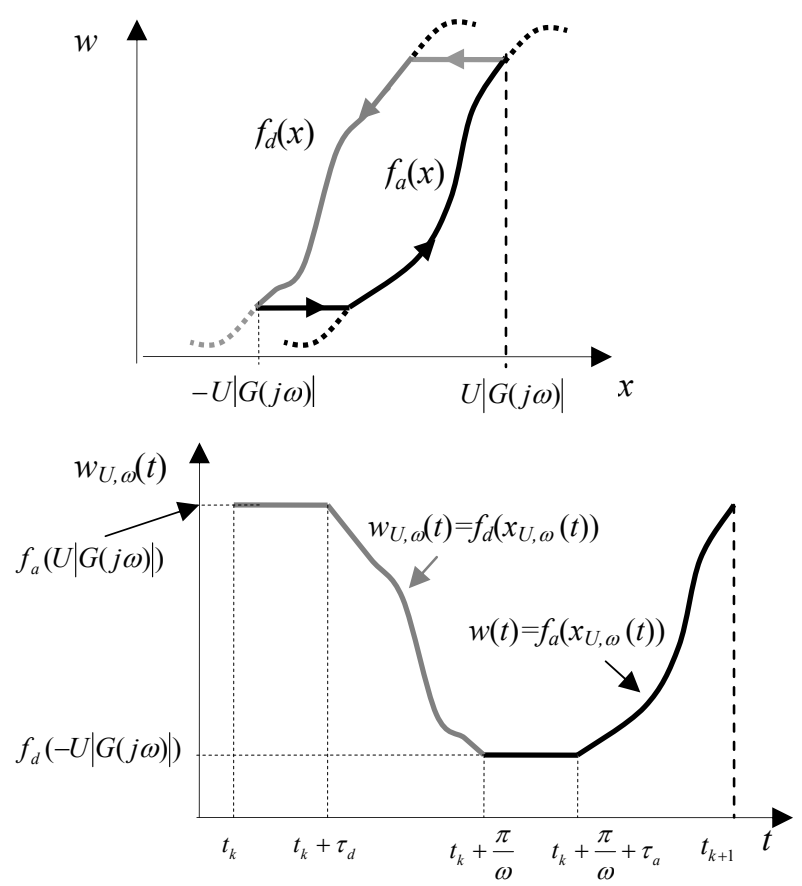

Fig. 2. Top: example of backlash limit cycle. Bottom: corresponding steady-state output $w_{U, \omega}(t)$ with constant stages making easier the identification of the backlash bordering functions $\left(f_{a}(x), f_{d}(x)\right)$ and the phase $\varphi(\omega)$.

Proposition 1. Consider the Wiener system described by equations (1)-(2) being excited by $u(t)=U \cos (\omega t)$.

1) Suppose $w_{U, \omega}(t)$ is time-varying and presents periodic constant stages and let $[\bar{t} \bar{t}+\bar{\tau})$ be one of the periodical intervals where $w_{U, \omega}(t)$ is constant. Then, $F[$.$] is a$ backlash, $\varphi(\omega)=\omega \bar{t}(\operatorname{modulo} \pi), \quad \bar{t} \in\left\{t_{k}, t_{k}+\frac{\pi}{\omega} ; k \in N\right\}$ and $\bar{t}+\bar{\tau} \in\left\{t_{k}+\tau_{d}, t_{k}+\frac{\pi}{\omega}+\tau_{a} ; k \in N\right\}$.

2) If $w_{U, \omega}(t)$ exhibits no constant stages then, $F[$.$] may be$ static or backlash, $f_{a}(U|G(j \omega)|)=f_{d}(U|G(j \omega)|)$, $f_{a}(-U|G(j \omega)|)=f_{d}(-U|G(j \omega)|)$ and $\tau_{a}=\tau_{d}=0$.

3) If $w_{U, \omega}(t)$ is all time constant, the couple $(U, \omega)$ is said to be nonadmissible. Admissibility can always be recovered by increasing $U$ provided $G(j \omega) \neq 0 \square$

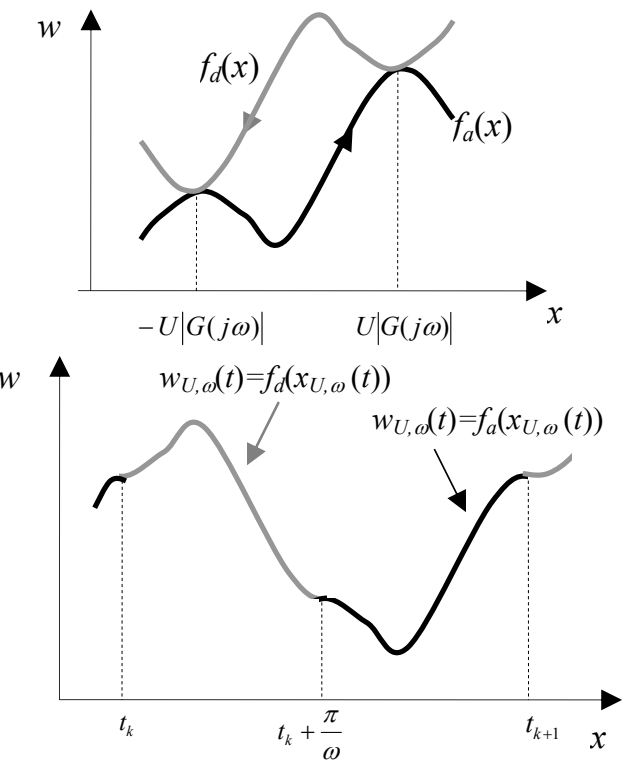

Fig. 3. Top: Backlash limit cycle with no horizontal segments. Bottom: corresponding output $w_{U, \omega}(t)$ with no constant stages, implying $\tau_{a}=\tau_{d}=0$.

\section{ESTIMATORS DESIGN}

\subsection{Relevant System Parameterizations}

The Wiener system (1)-(2) is excited by $u(t)=U \cos (\omega t)$ with $(U, \omega)$ admissible. To simplify estimators design, the resulting signal $w_{U, \omega}(t)$ is temporarily assumed to be measurable. Then, using (3) and (4a), one gets from (5b, 5e):

$$
\begin{aligned}
w_{U, \omega}(t) & =\sum_{i=0}^{n} a_{i}(U|G(j \omega)|)^{\mathrm{i}}(\cos (\omega t-\varphi(\omega)))^{i} \\
= & \sum_{i=0}^{n} \alpha_{i}^{*}(\cos (\omega t-\varphi(\omega)))^{i}, \\
& \text { for } t \in\left[t_{k}+\frac{\pi}{\omega}+\tau_{a} t_{k}+\frac{2 \pi}{\omega}\right) ; k \in N
\end{aligned}
$$

$$
\begin{aligned}
w_{U, \omega}(t)= & \sum_{i=0}^{n} d_{i}(U|G(j \omega)|)^{\mathrm{i}}(\cos (\omega t-\varphi(\omega)))^{i} \\
= & \sum_{i=0}^{n} \delta_{i}^{*}(\cos (\omega t-\varphi(\omega)))^{i} \\
& \quad \text { for } t \in\left[t_{k}+\tau_{d} t_{k}+\frac{\pi}{\omega}\right), k \in N
\end{aligned}
$$

where $\alpha_{i}^{*}$ and $\delta_{i}^{*}$ are lumped parameters defined by:

$\alpha_{i}^{*}=a_{i}(U|G(j \omega)|)^{i}, \delta_{i}^{*}=d_{i}(U|G(j \omega)|)^{i}, i=0 \ldots n$

Introduce the following auxiliary variable: 
$\sigma(\varphi(\omega), t)=\frac{1+\operatorname{sgn}(\sin (\omega t-\varphi(\omega)))}{2}$

Recall that the $t_{k}$ 's are such that, $\sin (\omega t-\varphi(\omega) \geq 0$

on

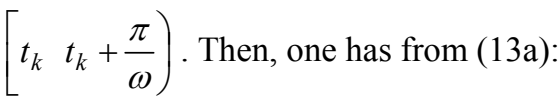

$\sigma(\varphi(\omega), t)=\left\{\begin{array}{l}1 \text { if } t_{k} \leq t<t_{k}+\pi / \omega \\ 0 \text { otherwise }\end{array}\right.$

Then, (12a-b) can be gathered into a single expression on $\left[t_{k}+\tau_{d} t_{k}+\frac{\pi}{\omega}\right) \cup\left[t_{k}+\frac{\pi}{\omega}+\tau_{a} t_{k}+\frac{2 \pi}{\omega}\right), k \in N:$

$$
\begin{gathered}
w_{U, \omega}(t)=\sum_{i=0}^{n} \alpha_{i}^{*}(1-\sigma(\varphi(\omega), t))(\cos (\omega t-\varphi(\omega)))^{i} \\
+\delta_{i}^{*} \sigma(\varphi(\omega), t)(\cos (\omega t-\varphi(\omega)))^{i} \\
=X^{T}(t, \varphi(\omega)) \theta^{*}
\end{gathered}
$$

where:

$$
\begin{aligned}
& \theta^{*}=\left[\begin{array}{llllll}
\alpha_{0}^{*} & \ldots & \alpha_{n}^{*} & \delta_{0}^{*} & \ldots & \delta_{n}^{*}
\end{array}\right]^{T} \\
& X^{T}(t, \varphi(\omega)) \\
& =\left[(1-\sigma(\varphi(\omega), t)) \quad \ldots(1-\sigma(\varphi(\omega), t))(\cos (\omega t-\varphi(\omega)))^{n}\right. \\
& \left.\sigma(\varphi(\omega), t) \quad \ldots \quad \sigma(\varphi(\omega), t)(\cos (\omega t-\varphi(\omega)))^{n}\right]
\end{aligned}
$$

Similar notations are associated to the model $\left(G^{-}(s), F^{-}[].\right)$:

$\theta^{-}=\left[\begin{array}{llllll}\alpha_{0}^{-} & \ldots & \alpha_{n}^{-} & \delta_{0}^{-} & \ldots & \delta_{n}^{-}\end{array}\right]^{T}$

$\alpha_{i}^{-}=a_{i}^{-}(U|G(j \omega)|)^{i}, \quad \delta_{i}^{-}=d_{i}^{-}(U|G(j \omega)|)^{i}$

where $i=0 \ldots n$. Recall that $\varphi^{-}(\omega) \stackrel{\text { def }}{=} \varphi(\omega)+\pi \quad$ (modulo $2 \pi)$. Then, it follows from (12a-b), using (11a):

$$
\begin{aligned}
w_{U, \omega}(t)=\sum_{i=0}^{n} \alpha_{i}^{-}\left(1-\sigma\left(\varphi^{-}(\omega), t\right)\right)\left(\cos \left(\omega t-\varphi^{-}(\omega)\right)\right)^{i} \\
\quad+\delta_{i}^{-} \sigma\left(\varphi^{-}(\omega), t\right)\left(\cos \left(\omega t-\varphi^{-}(\omega)\right)\right)^{i} \\
=\left(X^{T}\left(t, \varphi^{-}(\omega)\right)\right) \theta^{-}
\end{aligned}
$$

for $t \in\left[t_{k}^{-}+\tau_{d}^{-} t_{k}^{-}+\frac{\pi}{\omega}\right) \cup\left[t_{k}^{-}+\frac{\pi}{\omega}+\tau_{a}^{-} \quad t_{k}^{-}+\frac{2 \pi}{\omega}\right) \quad(k \in N)$.

To get benefit of (14b) and (17b) in the estimation of the lumped parameter vector $\theta^{*}$ or $\theta^{-}$, one needs:

i) an accurate estimator of $w_{U, \omega}$ (which is not measurable),

ii) three numbers $\bar{t}, \bar{\tau}_{a}, \bar{\tau}_{d}$ such that one of the two following inclusions holds, for some $k \in N$ :

$$
\begin{aligned}
& {\left[\bar{t}+\bar{\tau}_{d} \quad \bar{t}+\frac{\pi}{\omega}\right) \cup\left[\bar{t}+\frac{\pi}{\omega}+\bar{\tau}_{a} \quad \bar{t}+\frac{2 \pi}{\omega}\right)} \\
& =\left[t_{k}+\tau_{d} t_{k}+\frac{\pi}{\omega}\right) \cup\left[t_{k}+\frac{\pi}{\omega}+\tau_{a} t_{k}+\frac{2 \pi}{\omega}\right) \\
& {\left[\bar{t}+\bar{\tau}_{d} \bar{t}+\frac{\pi}{\omega}\right) \cup\left[\bar{t}+\frac{\pi}{\omega}+\bar{\tau}_{a} \bar{t}+\frac{2 \pi}{\omega}\right)} \\
& =\left[t_{k}^{-}+\tau_{d}^{-} \quad t_{k}^{-}+\frac{\pi}{\omega}\right) \cup\left[t_{k}^{-}+\frac{\pi}{\omega}+\tau_{a}^{-} \quad t_{k}^{-}+\frac{2 \pi}{\omega}\right]
\end{aligned}
$$

Based on Proposition 1, the second issue is coped with using the selection rule of Table 1.

Table 1. Selection of the Optimisation Set Parameters

1) If $w_{U, \omega}$ exhibits no periodic constant stages, let $\bar{\tau}_{a}=\bar{\tau}_{d}=0$ and $\bar{t}$ be any steady-state time.

2) a) If $w_{U, \omega}$ is varying but exhibits periodic constant stages (in steady-state) then, let $\bar{t}$ be any time when $w_{U, \omega}(t)$ begins a constant stage and let $\bar{t}+\bar{\tau}_{a}$ be the time when that stage ends.

b) If further $w_{U, \omega}(t)$ begins its next constant stage (after $\bar{t}$ ) at time $\bar{t}+\pi / \omega$ then, let $\bar{t}+\pi / \omega+\bar{\tau}_{d}$ denote the time when that stage ends. Otherwise, let $\bar{\tau}_{d}=0$.

\subsection{Relevant Cost functions}

We make use of the triplet $\left(\bar{t}, \bar{\tau}_{a}, \bar{\tau}_{d}\right)$, selected in Table 1 , to construct the optimization time domain $\left[\bar{t}+\bar{\tau}_{d} \quad \bar{t}+\frac{\pi}{\omega}\right) \cup\left[\bar{t}+\frac{\pi}{\omega}+\bar{\tau}_{a} \quad \bar{t}+\frac{2 \pi}{\omega}\right)$ which, by Proposition 1 , satisfies one of the two inclusions in (18a-b). Then, (14b) and (17b) suggest the following cost function:

$$
\begin{aligned}
J(\psi, \theta)=\int_{\bar{t}}+\frac{\bar{\omega}}{\bar{t}}+\frac{\pi}{\omega}\left(w_{U, \omega}(t)-X^{T}(t, \psi) \theta\right)^{2} d t \\
+\int_{\bar{t}+\frac{\pi}{\omega}+\bar{\tau}_{a}}^{\bar{t}+\frac{2 \pi}{\omega}}\left(w_{U, \omega}(t)-X^{T}(t, \psi) \theta\right)^{2} d t
\end{aligned}
$$

with $\psi \in[02 \pi) ; \theta \in \boldsymbol{R}^{2(n+1)}$. Equations (15a) and (16a) motivate the following structure of $\theta$ :

$$
\theta=\left[\begin{array}{llllll}
\alpha_{0} & \ldots & \alpha_{n} & \delta_{0} & \ldots & \delta_{n}
\end{array}\right]^{T}
$$

Proposition 2. Consider the Wiener system described by equations (1)-(2) being excited by $u(t)=U \cos (\omega t)$ with $(U, \omega)$ admissible. If $J(\psi, \theta)=0$ then, one has $(\psi, \theta)=\left(\varphi(\omega), \theta^{*}\right)$ or $(\psi, \theta)=\left(\varphi^{-}(\omega), \theta^{-}\right) \square$

Due to Proposition 2, an accurate estimate of $\left(\varphi(\omega), \theta^{*}\right)$ or $\left(\varphi^{-}(\omega), \theta^{-}\right)$can be obtained by minimizing $J(\psi, \theta)$. But, the latter involves $w_{U, \omega}(t)$ which is not accessible to measurements. Fortunately, an accurate estimator is available thanks to the steady-state periodic nature of $w_{U, \omega}$. It consists in the following periodical averaging, where $T=2 \pi / \omega$ :

$$
\begin{array}{ll}
\hat{w}_{U, \omega, N}(t)=\frac{1}{N} \sum_{k=1}^{N} y_{\omega}(t+k T) & \text { for } t \in[0 T) \\
\hat{w}_{U, \omega, N}(t+k T)=\hat{w}_{\omega}(t, N) & \text { for } k=1,2,3 \ldots
\end{array}
$$

The estimator (21a-b) was shown in (Giri et al., 2009) to be consistent. Now, substituting $\hat{w}_{U, \omega, N}(t)$ to $w_{U, \omega}(t)$ in (19), one gets the following cost function:

$\hat{J}(\psi, \theta, N)=\int_{\bar{t}_{N}+\bar{\tau}_{d, N}}^{\bar{t}_{N}+\frac{\pi}{\omega}}\left(\hat{w}_{U, \omega, N}(t)-X^{T}(t, \psi) \theta\right)^{2} d t$ 


$$
+\int_{\bar{t}_{N}+\frac{\pi}{\omega}+\bar{\tau}_{a, N}}^{\bar{t}_{N}+\frac{2 \pi}{\omega}}\left(\hat{\mathcal{w}}_{U, \omega, N}(t)-X^{T}(t, \psi) \theta\right)^{2} d t
$$

where $\bar{t}_{N}, \bar{\tau}_{a, N}, \bar{\tau}_{d, N}$ are selected using the rule of Table 1, replacing there $w_{U, \omega}(t)$ by $\hat{w}_{U, \omega, N}(t)$. As the latter is constructively periodic (with period $2 \pi / \omega$ ) over the overall interval $[0 \infty)$, the statement 'steady-state' in Table 1 then turns out to be useless when determining $\bar{t}_{N}, \bar{\tau}_{a, N}, \bar{\tau}_{d, N}$. Note that the later converge (w.p.1) to $\bar{t}, \bar{\tau}_{a}, \bar{\tau}_{d}$ because $\hat{w}_{U, \omega, N}$ is a consistent estimator of $w_{U, \omega}$. The cost function (22) will now be minimized with respect to the unknown quantities $(\varphi(\omega), \theta)$.

\subsection{Lumped Parameter and Phase Estimator}

The cost function $\hat{J}(\psi, \theta, N)$ is quadratic in $\theta$ but not in $\psi$. Nevertheless, we know by Proposition 1 (Part 1) that, if the output $\hat{w}_{\omega, N}$ exhibits constant stages then, $\varphi(\omega)=\omega \bar{t}$ (modulo $\pi)$. That is, letting $\psi=\omega \bar{t}$ in $\hat{J}(\psi, \theta, N)$ makes this quadratic in $\theta$ and allows the use of the standard leastsquares algorithm. This remark leads to the following estimator applicable when $\hat{w}_{\omega, N}$ exhibits constant stages:

$$
\begin{aligned}
& \hat{\varphi}_{L S}(\omega, N)=\omega \bar{t}_{N} \\
& \hat{\theta}_{L S}(N)=\left(\int_{I_{N}} X\left(t, \omega \bar{t}_{N}\right) X^{T}\left(t, \omega \bar{t}_{N}\right) d t\right)^{-1} \times \\
& \quad \int_{I_{N}} \hat{w}_{U, \omega, N}(t) X\left(t, \omega \bar{t}_{N}\right) d t
\end{aligned}
$$

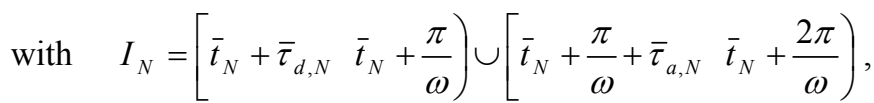
where ' $L S$ ' refers to 'least-squares'.

Now, let us focus on the more general case where $\hat{w}_{U, \omega, N}(t)$ exhibits no constant stages. By Table 1, one then has $\bar{t}_{N}=0$ and $\left(\bar{\tau}_{a, N}, \bar{\tau}_{d, N}\right)=(0,0)$. As there is no simple rule for determining $\varphi(\omega)$, the separable least-squares method is resorted to perform the minimization of $\hat{J}(\psi, \theta, N)$. Accordingly, $\psi$ is temporarily assumed to be known so that $\hat{J}(\psi, \theta, N)$ turns out to be a quadratic function in $\theta$. Then, the standard least-squares method gives the following estimate of $\theta$ :

$$
\theta(\psi, N)=\left(\int_{I_{N}} X(t, \psi) X^{T}(t, \psi) d t\right)^{-1} \int_{I_{N}} \hat{w}_{U, \omega, N}(t) X(t, \psi) d t
$$

Now, substituting the right sides of (24) to $\theta$ in (22) one gets a function in $\psi$ to be minimized. Specifically, one has:

$\hat{\varphi}_{S L S}(\omega, N)=\min _{0 \leq \psi \leq \pi} \hat{J}(\psi, \theta(\psi, N), N)$

where the index 'SLS' refers to 'separable least-squares'. Interestingly, the optimization problem (25a) is monodimensional and the search domain is a priori known $(0 \leq \psi \leq \pi)$. Therefore, the minimum should be relatively easy to find using e.g. a graphical search method. Once
$\hat{\varphi}_{S L S}(\omega, N)$ is obtained, it is substituted to $\psi$ in (24) yielding the following estimator of $\theta$ :

$\hat{\theta}_{S L S}(N)=\left(\int_{I_{N}} X\left(t, \hat{\varphi}_{S L S}(\omega, N)\right) X^{T}\left(t, \hat{\varphi}_{S L S}(\omega, N)\right) d t\right)^{-1} \times$

$$
\int_{I_{N}} \hat{w}_{U, \omega, N}(t) X\left(t, \hat{\varphi}_{S L S}(\omega, N)\right) d t
$$

Using (23a-b) and (25a-b), one gets the following phase and lumped parameter estimator:

$(\hat{\varphi}, \hat{\theta})=\left\{\begin{array}{c}\left(\hat{\varphi}_{L S}, \hat{\theta}_{L S}\right) \text { if }\left(\bar{\tau}_{a, N}, \bar{\tau}_{d, N}\right) \neq(0,0) \\ \left(\hat{\varphi}_{S L S}, \hat{\theta}_{S L S}\right) \text { if }\left(\bar{\tau}_{a, N}, \bar{\tau}_{d, N}\right)=(0,0)\end{array}\right.$

Proposition 3. Let the Wiener system (1)-(2) excited by $u(t)=U \cos (\omega t)$. Then, the estimator $(\hat{\varphi}, \hat{\theta})$ defined by (26) is consistent in the sense that $(\hat{\varphi}(\omega, N), \hat{\theta}(N))$ converges to $\left\{\left(\varphi(\omega), \theta^{*}\right),\left(\varphi^{-}(\omega), \theta^{-}\right)\right\}$

\subsection{Estimators for Nonlinearity Border Coefficients and Frequency Gain Modulus}

Equation (20) motivates the following notations:

$\hat{\theta}(N)=\left[\begin{array}{llllll}\hat{\alpha}_{0}(N) & \ldots & \hat{\alpha}_{n}(N) & \hat{\delta}_{0}(N) & \ldots & \hat{\delta}_{n}(N)\end{array}\right]^{T}$

Now, the question is how to recover the frequency gain modulus $|G(j \omega)|$ and the coefficients, $\left(a_{i}, d_{i} ; i=1 \ldots n\right)$ or $\left.\left(a_{i}^{-}, d_{i}^{-} ; i=1 \ldots n\right)\right)$, of the bordering functions $\left(f_{a}(x), f_{d}(x)\right)$ or $\left(f_{a}^{-}(x), f_{a}^{-}(x)\right)$. The starting point is the couple of equations (12c) and (16b) which relate the coefficients of the bordering polynomials to the lumped parameters. Accordingly, one immediately gets:

$a_{0}=\alpha_{0}^{*}, d_{0}=\delta_{0}^{*}$ and $a_{0}^{-}=\alpha_{0}^{-}, d_{0}^{-}=\delta_{0}^{-}$

The remaining coefficients, $\left(a_{i}, b_{i}\right)$ and $\left(a_{i}^{-}, b_{i}^{-}\right)$ $(i=1 \ldots m)$, can not be uniquely determined, because the quantity $|G(j \omega)|$ is also unknown. At this point, consider the re-scaled model $(G(s) / K, F[K x])$ and note that $F[K x]$ is also a backlash bordered by polynomials $\left(f_{a}(K x), f_{d}(K x)\right)$ with coefficients $\left(\bar{a}_{0}, \bar{a}_{1}, \ldots, \bar{a}_{n}\right) \stackrel{\text { def }}{=}\left(a_{0}, a_{1} K, \ldots, a_{n} K^{n}\right)$ and $\left(\bar{d}_{0}, \bar{d}_{1}, \ldots, \bar{d}_{n}\right)^{\text {def }}=\left(d_{0}, d_{1} K, \ldots, d_{n} K^{n}\right)$. These expressions suggest that one convenient choice of the scaling factor is:

$K=\left(\sum_{i=1}^{n}\left|a_{i}\right|^{n / i}\right)^{-1 / n}$

implying $\sum_{i=1}^{n}\left|\bar{a}_{i}\right|^{n / i}=1$. To avoid introducing additional notations, the re-scaled model $(G(s) / K, F[K x])$, will still to be denoted $(G(s), F[x])$. It turns out that, this model and its dual $\left(G^{-}(s), F^{-}[x]\right)$ are the only that feature the properties: $\sum_{i=1}^{n}\left|a_{i}\right|^{n / i}=1, \quad \sum_{i=1}^{n}\left|a_{i}^{-}\right|^{n / i}=1$

Using (30a), it readily follows from (12c) and (16b) that: 


$$
\begin{aligned}
\left|\alpha_{i}^{*}\right|^{n / i}=\left|a_{i}\right|^{n / i}(U|G(j \omega)|)^{n} ; \quad\left|\alpha_{i}^{-}\right|^{n / i}= & \left|a_{i}^{-}\right|^{n / i}(U|G(j \omega)|)^{n} \\
& (i=1 \ldots n)
\end{aligned}
$$

Adding both sides of the first (resp. second), equality in (30b) over $i=1 \ldots m$, yields using (30a):

$\sum_{i=1}^{n}\left|\alpha_{i}^{*}\right|^{n / i}=\sum_{i=1}^{n}\left|a_{i}\right|^{n / i}\left(U_{1}|G(j \omega)|\right)^{n}=(U|G(j \omega)|)^{n}$

$\sum_{i=1}^{n}\left|\alpha_{i}^{-}\right|^{n / i}=\sum_{i=1}^{n}\left|a_{i}^{-}\right|^{n / i}(U|G(j \omega)|)^{n}=(U|G(j \omega)|)^{n}$

From (31a-b) one immediately gets:

$|G(j \omega)|=\frac{1}{U}\left(\sum_{i=1}^{n}\left|\alpha_{i}^{*}\right|^{n / i}\right)^{1 / n}=\frac{1}{U}\left(\sum_{i=1}^{n}\left|\alpha_{i}^{-}\right|^{n / i}\right)^{1 / n}$

This uniquely determines the frequency gain modulus $|G(j \omega)|$ in terms of both sets of lumped parameters $\left\{\alpha_{i} ; i=1 \ldots n\right\}$ and $\left\{\alpha_{i}^{-} ; i=1 \ldots n\right\}$. Using (32), one gets from (13c) and (16b), respectively:

$$
\begin{aligned}
& \alpha_{i}^{*}=a_{i}\left(\sum_{i=1}^{n}\left|\alpha_{i}^{*}\right|^{n / i}\right)^{i / n}, \delta_{i}^{*}=d_{i}\left(\sum_{i=1}^{n}\left|\alpha_{i}^{*}\right|^{n / i}\right)^{i / n} \\
& \alpha_{i}^{-}=a_{i}^{-}\left(\sum_{i=1}^{n}\left|\alpha_{i}^{-}\right|^{n / i}\right)^{i / n}, \delta_{i}^{-}=d_{i}^{-}\left(\sum_{i=1}^{n}\left|\alpha_{i}^{-}\right|^{n / i}\right)^{i / n}
\end{aligned}
$$

with $i=1 \ldots n$. From the above expressions one easily gets:

$$
\begin{aligned}
& a_{i}=\frac{\alpha_{i}^{*}}{\left(\sum_{i=1}^{n}\left|\alpha_{i}^{*}\right|^{n / i}\right)^{i / n}}, d_{i}=\frac{\delta_{i}^{*}}{\left(\sum_{i=1}^{n}\left|\alpha_{i}^{*}\right|^{n / i}\right)^{i / n}} \\
& a_{i}^{-}=\frac{\alpha_{i}^{-}}{\left(\sum_{i=1}^{n}\left|\alpha_{i}^{-}\right|^{n / i}\right)^{i / n}}, d_{i}^{-}=\frac{\delta_{i}^{-}}{\left(\sum_{i=1}^{n}\left|\alpha_{i}^{-}\right|^{n / i}\right)^{i / n}}
\end{aligned}
$$

with $i=1 \ldots n$. Equations (27), (28), (32) and (34a-b) suggest the following estimators for the frequency gain modulus and the coefficients of bordering functions:

$$
\begin{aligned}
& \left|\hat{G}_{N}(j \omega)\right|=\frac{1}{U}\left(\sum_{i=1}^{n}\left|\hat{\alpha}_{i}(N)\right|^{n / i}\right)^{1 / n} \\
& \hat{a}_{0}(N)=\hat{\alpha}_{0}(N), \quad \hat{a}_{i}(N)=\frac{\hat{\alpha}_{i}(N)}{\left(\sum_{i=1}^{n}\left|\hat{\alpha}_{i}(N)\right|^{n / i}\right)^{i / n}} \\
& \hat{d}_{0}(N)=\hat{\delta}_{0}(N), \quad \hat{d}_{i}(N)=\frac{\hat{\delta}_{i}(N)}{\left(\sum_{i=1}^{n}\left|\hat{\delta}_{i}(N)\right|^{n / i}\right)^{i / n}}
\end{aligned}
$$

where $i=1 \ldots n$.

Proposition 4. The estimators (35)-(37) are consistent in the sense that, one has (w.p.1 as $N \rightarrow \infty$ ): $\left|\hat{G}_{N}(j \omega)\right|$ converges to $|G(j \omega)|$ and $\left(\hat{a}_{i}(N), \hat{d}_{i}(N) ; i=0 \ldots n\right)$ converges to $\left\{\left(a_{i}, d_{i} ; i=0 \ldots n\right),\left(a_{i}^{-}, d_{i}^{-} ; i=0 \ldots n\right)\right\} \square$

\section{CONCLUSIONS}

The problem of Wiener system identification has been addressed in the case where the system nonlinear element is a backlash bordered by polynomial lines. These are allowed to be noninvertible and crossing, making possible to account for static noninvertible nonlinearities. To our knowledge, no previous works have considered this class of Wiener systems. The problem is coped with in the frequency-domain using the identification method, described in Section 4, involving standard and separable least-squares estimators. All estimators are shown to be consistent.

\section{SIMULATION}

The simulation results have been removed due to space limitation. They will be presented at the conference.

\section{REFERENCES}

Bai E.W. and J. Reyland, (2009). Towards identification of Wiener systems with the least amount of a priori information: IIR cases. Automatica, 45, 956-964.

Bruls, J., C.T. Chou, B.R.J. Heverkamp and M. Verhaegen (1999). Linear and nonlinear system identification using separable least squares, European J. Control, 5, 116-128.

Chen, H.F. (2006). Recursive identification for Wiener model with discontinuous piece-wise linear function, IEEE Transactions on Automatic Control, 51, 390-400.

Giri, F., Y. Rochdi and F.Z. Chaoui, (2009). An analytic geometry approach to Wiener system frequency identification, IEEE Trans. Aut. Control, 54, 683-696.

Giri F., Y. Rochdi, A. Brouri, F.Z. Chaoui (2010). Frequency identification of Wiener systems with hard backlash nonlinearity. IFAC Symposium on Nonlinear Control Systems, Italy.

Greblicki W., M. Pawlak, Non-Parametric System Identification. Cambridge University Press, 2008

$\mathrm{Hu}$ X.L. and H.F. Chen (2005). Strong consistence of recursive identification for Wiener systems. Automatica, 41, 1905-1916.

Mzyk G. (2010). Stochastic Identification of Wiener Systems. In: Block-oriented nonlinear system identification (F. Giri and E.W. Bai (Ed)). Springer, U.K.

Vanbaylen L., R. Pintelon, and J. Schoukens (2009). Blind maximum-likelihood identification of wiener systems. IEEE Trans. Signal Processing, 57, 3017-3029.

Vörös J., (1997). Parameter identification of Wiener systems with discontinuous nonlinearities. Systems and Control Letters, 44, 363-372.

Wills A. and L. Ljung, (2010). Wiener system identification using the maximum likelihood method. In: Blockoriented nonlinear system identification (Giri F. and E.W. Bai, (Ed)). Springer, U.K.

Westwick D. and M. Verhaegen (1996). Identifying MIMO Wiener systems using subspace model identification methods. Signal Processing, 52, 235-258. 